\title{
LETTER
}

\section{IL-6 may be a good biomarker for earlier detection of COVID-19 progression}

\author{
Changsong Wang ${ }^{1,2}$, Dongsheng Fei ${ }^{1}$, Xueting $\mathrm{Li}^{1,2}$, Mingyan Zhao ${ }^{1 *}$ and Kaijiang $\mathrm{Yu}^{{ }^{1 *}}$ (D)
}

๑ 2020 Springer-Verlag GmbH Germany, part of Springer Nature

\section{Dear Editor,}

The global outbreak of COVID-19 has seriously endangered healthcare systems in China and worldwide. 15.7\% of cases of COVID-19 were severe, and 5\% were critical according to guidelines on the diagnosis and treatment of new coronavirus pneumonia (version 6) [1]. One recent study reported that the fatality rate of COVID-19 in China is $1.4 \%$ [2]. Despite showing a lower case fatality rate, COVID-19 has so far resulted in more deaths than SARS and MERS combined [3, 4]. It is important to establish whether there is a difference between severe and critical patients, as this might help us to identify critical patients in a more timely and effective manner. However, to date, few biomarkers of progression of COVID-19 have been reported.

In this single-center retrospective study, written informed consent was duly obtained from all patients. The study was approved by the Ethics Committee of the first affiliated hospital of Harbin Medical University. A total of 45 COVID-19 patients were included. Our study defined the severity of COVID-19 according to the guidelines on the diagnosis and treatment of new coronavirus pneumonia (version 6). We also attempted to evaluate the severity of COVID-19 patients using the sequential organ failure assessment (SOFA) scoring system.

A logistic regression model was used to analyze the factors influencing the severity of the disease. Among the total of 19 adjustable variables (seen in supplementary data) in the univariate analysis, 3 and 2 significant

\footnotetext{
*Correspondence: Ixt886@163.com; kaijiangyu002@163.com

${ }^{1}$ Department of Critical Care Medicine, The First Affiliated Hospital of Harbin Medical University, Harbin Medical University, Harbin 150081 Heilongjiang, China

Full author information is available at the end of the article

Changsong Wang, Dongsheng Fei and Xueting Li have contributed equally to this work.
}

indicators were found two different evaluation systems, respectively. In addition to using these indicators, comorbidities, age, and sex were also included in the model for multivariate regression analysis.

The patients' baseline characteristics were compared between the severe and the critical group according to the two different evaluation systems. Classification according to version 6 (eTable-1): among 33 severe patients (mean age $63.13 \pm 14.1$ years), 17 [52\%] were male; among 12 critical patients mean age (62.58 \pm 9.89 years), $6[50 \%]$ were male. IL-6 levels (Fig. 1a) $(p=0.0004)$ and IL-10 levels (eFig. 2) $(p=0.0073)$ were significantly lower in the severe group than in the critical group (eTable 1); the results showed that IL-6 levels (Fig. 1b, eTable 2-4) were related to the severity of COVID patients $(p=0.0121)$. The AUC of IL- 6 was 0.848 , the sensitivity $90.91 \%$, the specificity $66.67 \%$, and the cutoff value $19.03 \mathrm{pg} / \mathrm{ml}$. Classification according to the SOFA score system (Severe: SOFA <2; Critical: SOFA $\geq 2$ ) (eTable 5): among 14 severe patients (mean age $57.69 \pm 19.34$ years), 8 [57\%] were male; among 31 critical patients (mean age $65.19 \pm 8.63$ years), 15 [48\%] were male. Lower IL-6 levels (Fig. 1c) $(p<0.0001)$ and higher CD3 (eFig. 3) $(p=0.0032)$ were found in the severe group compared with the critical group. The results (see supplementary data) showed that IL-6 levels (Fig. 1d, eTable 6-8) were related to the severity of COVID patients $(p=0.0151)$. The AUC of IL- 6 was 0.910 , the sensitivity $85.71 \%$, the specificity $96.77 \%$, and the cutoff value $6.11 \mathrm{pg} / \mathrm{ml}$.

In conclusion, up-regulated IL-6 levels may serve as a potential marker for predicting progression of COVID19 patients. Whether evaluated according to the version 6 guidelines or the SOFA score, IL-6 is good at assessing COVID-19 progression. 

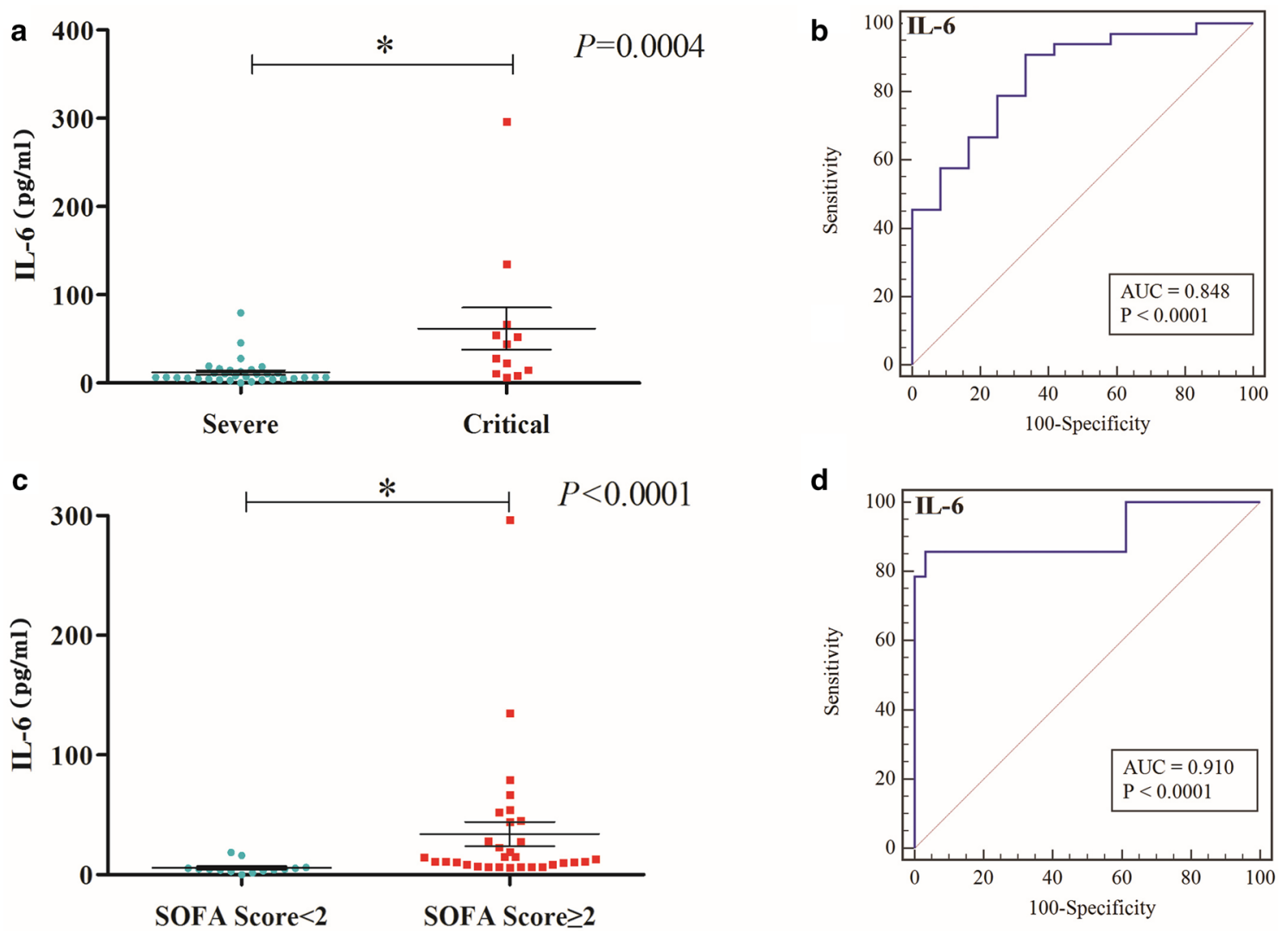

Fig. $1 \mathrm{LL}-6$ levels $(p=0.0004)$ a was significantly lower in severe group than critical group due to Guidelines of the Diagnosis and Treatment of New Coronavirus Pneumonia (version 6), AUC of IL-6 is 0.848 , sensitivity is $90.91 \%$, specificity is $66.67 \%$ (b); IL-6 levels $(p<0.0001)$, c was significantly different in two disease groups due to SOFA scoring system., AUC of IL-6 is 0.910 , sensitivity is $85.71 \%$, specificity is $96.77 \%$ (d)

\section{Electronic supplementary material}

The online version of this article (https://doi.org/10.1007/s00134-020-06065-8) contains supplementary material, which is available to authorized users.

\section{Author details}

${ }^{1}$ Department of Critical Care Medicine, The First Affiliated Hospital of Harbin Medical University, Harbin Medical University, Harbin 150081, Heilongjiang, China. ${ }^{2}$ Department of Critical Care Medicine, Harbin Medical University Cancer Hospital, Harbin Medical University, Harbin 150081, Heilongjiang, China.

\section{Acknowledgements}

Sincere thanks to all the staff of Heilongjiang COVID-19 critical patients treatment center. Thank you for your bravery and dedication.

\section{Funding}

Supported by Novel coronavirus pneumonia emergency treatment and diagnosis technology research project of Heilongjiang provincial science and Technology Department, the National Natural Science Foundation of China (Nos. 81571871, 81770276), Nn10 program of Harbin Medical University Cancer Hospital.

\section{Compliance with ethical standards}

\section{Conflicts of interest}

On behalf of all authors, the corresponding author states that there is no conflict of interest.

\section{Publisher's Note}

Springer Nature remains neutral with regard to jurisdictional claims in published maps and institutional affiliations.

\section{Accepted: 18 April 2020}

Published online: 8 May 2020

\section{Reference}

1. Mahase E (2020) Coronavirus covid-19 has killed more people than SARS and MERS combined, despite lower case fatality rate. BMJ 368:m641

2. Guan WJ, Ni ZY, Hu Y, Liang WH, Ou CQ, He JX, Liu L, Shan H, Lei CL, Hui DSC, Du B, Li LJ, Zeng G, Yuen KY, Chen RC, Tang CL, Wang T, Chen PY, Xiang J, Li SY, Wang JL, Liang ZJ, Peng YX, Wei L, Liu Y, Hu YH, Peng P, Wang JM, Liu JY, Chen Z, Li G, Zheng ZJ, Qiu SQ, Luo J, Ye CJ, Zhu SY, Zhong NS (2020) Clinical Characteristics of Coronavirus Disease 2019 in China. N Engl J Med. https://doi.org/10.1056/NEJMoa2002032

3. Centers for Disease Control and Prevention (20050724) Frequently asked questions about SARS. https://www.cdc.gov/sars/about/faq.html. Accessed 24 July 2019

4. World Health Organization (20191130). Middle East respiratory syndrome coronavirus (MERS-CoV). https://www.who.int/emergencies/mers-cov/ en. Accessed 30 Nov 2019 